S. Kwok, M. Dopita, and R. Sutherland, eds.

\title{
HST Measurements of the Angular Expansion, Kinematics and Distance of the Planetary Nebula $\mathrm{BD}+30^{\circ} 3639$
}

\section{J. Patrick Harrington, Jianyang Li}

Dept. of Astronomy, University of Maryland, College Park, MD, USA

Kazimierz J. Borkowski

Dept. of Physics, North Carolina State University, Raleigh, NC, USA

Abstract. The HST WFPC2 camera was used to obtain images of the planetary nebula $\mathrm{BD}+30^{\circ} 3639$ at two epochs separated by 5.66 years. The expansion of the nebula in $\mathrm{H} \alpha$ and [N II] has been measured using several methods. Detailed expansion maps for both emission lines were constructed from nearly 200 almost independent features. There is good agreement between the (independent) $\mathrm{H} \alpha$ and [N II] proper motions. There are clear deviations from uniform radial expansion, with higher expansion rates in regions where the shell is faintest, such as the south-west quadrant.

HST STIS echelle spectra obtained in the C II] $\lambda 2326$ multiplet provide well-resolved expansion velocities at two position angles. We find that the central velocity split is $\pm 36.3 \mathrm{~km} \mathrm{~s}^{-1}$ at a position angle of $99^{\circ}$.

To determine the distance of $\mathrm{BD}+30^{\circ} 3639$ by comparison of the angular expansion and the radial expansion, we must address the problem of the three dimensional shape of the nebula. We measured the angular expansion along the position of the $99^{\circ}$ echelle slit, finding displacements of $4.25 \mathrm{mas} \mathrm{yr}^{-1}$ at the shell edge ( $2^{\prime \prime} 47$ from the center). If the nebula were spherical, this would imply a distance of $1.80 \mathrm{kpc}$. But there is evidence that the nebula is elongated along the line of sight, which suggests that the actual distance is less. Radio continuum images from 5 and 15 $\mathrm{GHz}$ VLA observations provide information on the extent of the radial elongation. We fit the radio brightness variation and the echelle data by approximating the nebula as an ellipsoid. Our model has an axial ratio of 1.56 , is inclined to the line of sight by $9: 7$, and exhibits an expansion in the plane of the sky which is $2 / 3$ that in the radial direction, leading to a distance of $1.2 \mathrm{kpc}$. Based on a recent model atmosphere (Crowther et al., these proceedings), this distance implies a stellar luminosity of $4250 \mathrm{~L}_{\odot}$. 